\title{
Adjunction of Avidin to a Cysteamine Self-Assembled Monolayer for Impedimetric Immunosensor
}

\author{
Oussama Ouerghi'1,2*, M. Fethi Diouani², A. Belkacem¹, A. Elsanousi' \\ Nicole Jaffrezic-Renault ${ }^{3}$ \\ ${ }^{1}$ Prince Sattam Bin Abul Aziz University, AlKharj, Kingdom of Saudi Arabia \\ ${ }^{2}$ Laboratory of Epidemiology and Veterinary Microbiology (LR11IPT03,) Pasteur Institute of Tunis, Tunis, Tunisia \\ ${ }^{3}$ Institute of Analytical Sciences, University of Lyon, UMR CNRS 5280, Villeurbanne, France \\ Email: "O.Ouerghi@sau.edu.sa,"oussamaouerghi@yaho.com
}

Received 5 October 2015; accepted 1 January 2016; published 4 January 2016

Copyright (C) 2016 by authors and Scientific Research Publishing Inc.

This work is licensed under the Creative Commons Attribution International License (CC BY).

http://creativecommons.org/licenses/by/4.0/

(c) (i) Open Access

\begin{abstract}
In this work an impedimetric immunosensor based on affinity immobilization method of a biotin labelled anti-human IgG antibody, used as a model system, was reported. The experimental procedure involves the growth of a self-assembled monolayer of a thiol (cysteamine) carrying terminal amine groups on gold electrodes. Glutarardehyde, a homobifunctional cross-linker, was used as a coupling reagent for the covalent linking of avidin to the amine groups of cysteamine. The attachment of the biotin labeled antibodies (anti-Human IgG) to the subsequent modified gold electrode was achieved by affinity interactions tacking advantage of the strong avidin-biotin bridge. The stepwise assembly process of the electrode was interrogated by means of cyclic voltammetry, electrochemical impedance spectroscopy and contact angle measurements. The response of the antibody modified electrode to their target antigens was investigated in the presence of BSA (bovin serum albumin) in order to alleviate non-specific adsorption problems. A proposed electrical model was used to analyse the experimental data. The resulting immunosensor has a linear dynamic range of $100-900 \mathrm{ng} \cdot \mathrm{ml}^{-1}$ of antigen and a detection limit of 100 $\mathbf{n g} \cdot \mathbf{m l}^{-1}$.
\end{abstract}

\section{Keywords}

Avidin, Cysteamine, Electrochemical Impedance Spectroscopy, Immunosensor

\footnotetext{
${ }^{*}$ Corresponding author.
}

How to cite this paper: Ouerghi, O., Diouani, M.F., Belkacem, A., Elsanousi, A. and Jaffrezic-Renault, N. (2016) Adjunction of Avidin to a Cysteamine Self-Assembled Monolayer for Impedimetric Immunosensor. Journal of Biomaterials and Nanobiotechnology, 7, 1-12. http://dx.doi.org/10.4236/ibnb.2016.71001 


\section{Introduction}

For three decades, impedimetric immunosensors have been the subject of increasing interest as evidenced by the large number of reviewed published papers [1]-[5]. Basically, electrochemical impedance spectroscopy EIS is an alternative current technique that describes the response of an electrochemical cell to a small amplitude AC potential difference over imposed on a DC bias potential. The resulting current changes in time with respect to the perturbing voltage, and the ratio $\mathrm{V}(\mathrm{t}) / \mathrm{I}(\mathrm{t})$ is defined as the impedance $(\mathrm{Z})$. Regularly $10 \mathrm{mV}$ in amplitude or less was applied for several reasons. First, the current-voltage relationship is often linear only for small perturbations [6] [7], and only in this regime impedance is defined. A second reason for using a small excitation signal is to avoid the denaturation biorecognition element. Thus, appropriately performed, electrochemical impedance spectroscopy does not damage the biomolecular probe layer, which is an important advantage over voltammetry and amperometry measurements where higher voltages are often applied. Furthermore, it is also considered as a label free technique as it doesn’t require any special labelling [3].

However, it is recognizable that the limits of label-free impedimetric immunosensor performances are more often fixed by the affinity step, attributed to the high specificity of immune interactions, than by the readout step itself. This suggests the need for further research efforts in bimolecular probe immobilization chemistry in addition to the minimization of non-specific binding. More particularly, the recognition immune species immobilization is considered to be the most important prerequisite for the fabrication of impedimetric immunosensor. For this reason, the key criteria during the immobilization process are how to fully maintain the immune probe, namely antibody or antigen, conformation and activity. Typically, the immune probe can be immobilized to the electrode surface either by covalent attachment or by affinity interactions [8] [9]. Although covalent binding provides a very stable recognition immune-layer, it can likely disrupt the immobilized proteins which in return drastically affect the performance of the derivative immunosensor. To overcome this critical drawback caused by covalent immobilization, affinity methods appear to be a promising alternative, as they ensure a high orientation of the immune probe without alteration of its activity [10]. Previously, IgG antibodies were directly attached to a cyano-terminated electro polymerized polypyrrole on gold electrode. The immobilization was achieved by electrostatic interaction between the amine groups on the Fc part of antibodies and the cyano terminal groups of the polymer [11]. Alternatively, proteins A and G, commonly used in affinity chromatography for antibody purification, were used to immobilize immunoglobulins IgG through their Fc region on the immunosensor electrode surface [12]. The IgG antibodies are attached to the electrode in a site-directed manner with their antigen-binding regions directed towards the analyte. Among the different affinity immobilization methods, immobilization based on biotin-avidin system for the binding of biotin labelled probes, has aroused considerable attention, as it contributes to improve significantly the immunosensors sensitivity and stability [13] [14]. Indeed, avidin-biotin bond formation is rapid and is usually unaffected by temperature, organic solvents and $\mathrm{pH}$ [14]. This method proved to have high control over the molecule orientation, which is an important requirement for the improvement of the immunosensor characteristics. However the major weakness of this method is the inevitability to label proteins with biotin prior to immobilization. Such immobilization strategy was already used by several groups to attach proteins to electrodeposited biotinylated polypyrrole film [15]-[20].

Avidin, found in hens egg-whites, is a very fascinating protein because of its extraordinarily high binding affinity for biotin or biotin conjugated proteins. In fact, the biotin-avidin interaction is one of the strongest non-covalent interactions (affinity constant $\mathrm{K}_{\mathrm{a}} \sim 10^{15} \mathrm{M}^{-1}$ ) [21]. Commonly, avidin was associated to biotin labelled polymer generated on the surface of solid electrodes either by electroplymerization or by self-assembling in order to immobilize biotin conjugate proteins [22].

On the other hand, self-assembled monolayer (SAM) of different thiol chains has been intensively used as platform for the development of biosensors [23]. SAMs offer the possibility to get ultrathin organic film of controlled thickness and specific terminal functional groups requested for the immobilization of individual biomolecules [24]-[26]. Particularly, the modification of gold electrodes by simple SAM using cysteamine was widely used for the immobilization of proteins such as enzymes or antibodies [27]-[30]. Indeed, a label free impedimetric immunosensor for the detection of cancer biomarkers was developed, by covalent immobilization of specific antibody to cysteamine self-assembled monolayer on Au electrode surface using diisothiocyanate as a crosslinker [12]. Likewise, self-assembled cysteamine monolayer on Au electrode surface was used for direct non covalent immobilization of glucose oxidase in order to develop glucose biosensor [31]. Earlier, a QCM based biosensor for E. coli was developed via covalent immobilization of polyclonal antibodies onto gold-coated QCM quartz crystals via a cysteamine self-assembled monolayer using glutaraldehyde as a cross-linker [32]. 
In this work, gold bare electrode was first modified by cysteamine. The amine-terminated SAMs modified electrodes were next functionalized with avidin using glutaraldehyde as a homobifunctional crosslinker. Biotinylated IgG antiboby (biotin conjugate human IgG) was then anchored to avidin via the formation of avidin biotin bridge. The different steps of the immunosensor fabrication were investigated by cyclic voltammetry, electrochemical impedance spectroscopy and contact angle measurements. Moreover, to the best of our knowledge, this is the first report based on the covalent immobilization of avidin on a self-assembled monolayer of cysteamine.

\section{Experimental}

\subsection{Materials}

Cysteamine: $\mathrm{HS}\left(\mathrm{CH}_{2}\right)_{2} \mathrm{NH}_{2} \cdot \mathrm{HCl}$ (from Fluka), bovine serum albumin (BSA) (Sigma-Aldrich), glutaraldehyde: $\mathrm{OHC}\left(\mathrm{CH}_{2}\right)_{3} \mathrm{CHO}$ (Sigma-Aldrich) were used to prepare the immunosensing surface. The antibody used was polyclonal anti-human IgG biotin conjugate developed in goat purchased from Sigma. The antigen was a human IgG reagent grade, from serum, purchased from Sigma. The supporting electrolyte was a phosphate buffer solution (PBS), prepared by dissolving one tablet of phosphate buffer saline, purchased from Sigma, in $200 \mathrm{ml}$ of deionized water. The obtained solution consisted of a $10 \mathrm{mM}$ phosphate buffer, $2.7 \mathrm{mM}$ potassium chloride, and $137 \mathrm{mM}$ sodium chloride. The $\mathrm{pH}$ of the solution at $25^{\circ} \mathrm{C}$ was 7.4.

\subsection{Antibody Immobilization Procedure}

First, the gold plate electrodes (99.99\% pure) were cleaned for about $10 \mathrm{~min}$ in an etching solution: $1 / 4\left(\mathrm{H}_{2} \mathrm{O}_{2}\right.$ 35\%) $3 / 4\left(\mathrm{H}_{2} \mathrm{SO}_{4} 96 \%\right)$ at room temperature. The pretreated electrodes were immediately, thoroughly rinsed with deionized water in order to remove any deposited impurities, and then dried under a stream of nitrogen. Clean gold-plate electrodes were immersed in a $10 \mathrm{mM}$ cysteamine in absolute ethanol solution overnight at room temperature. After rinsing with deionized water and drying with nitrogen, the gold plates were mounted inside the electrochemical cell. Next, a 2\% solution of glutaraldehyde in PBS was injected into the cell for 30 min. Glutaraldehyde is a reactive crosslinking agent that binds amine groups located on cysteamine. This was followed by a PBS rinse to remove any unreacted glutaraldehyde from the electrode surface. Then, a $2 \mathrm{mg} \cdot \mathrm{ml}^{-1}$ of avidin solution was injected for $30 \mathrm{~min}$ at $5^{\circ} \mathrm{C}$ then followed by an excessive rinsing with deionized water to remove unreacted avidin molecules. Immediately after that, a solution of anti-human antibodies $(10 \mu \mathrm{g} / \mathrm{mL}) \mathrm{in}$ PBS was injected into the cell for $60 \mathrm{~min}$. This step was followed by a PBS rinse to remove unbound antibody. Next, a BSA solution in PBS $(10 \mathrm{mg} / \mathrm{mL})$ was injected for 60 min to block the unreacted sites. A final PBS rinse was applied to remove unbounded BSA. The prepared electrode was then ready for the detection of the target antigen.

\subsection{Contact Angle Measurements}

Contact angles were obtained using the sessile drop method with a GBX Scientific Instrument (Romans, France). The drop image was stored by a video camera and an image analysis system calculated the contact angle $(\theta)$ from the shape of the drop. Millipore water was used as a probe for contact angle calculations. For each sample, 4 drops were analysed on each substrate. The drop volume was fixed to be about $2 \mu \mathrm{l}$ in order to neglect the gravity effect and therefore to highlight only the interfacial phenomena. The results were processed using Windrop $^{2+}$ software.

\subsection{Electrochemical Measurements}

The electrochemical measurements were performed in a conventional electrochemical cell, with three electrodes and a volume of $3.5 \mathrm{ml}$. The counter electrode was a platinum plate with an active surface area of $0.48 \mathrm{~cm}^{2}$, whereas the active surface area of the working gold plate electrode, fixed by an O-ring seal, was $0.16 \mathrm{~cm}^{2}$. The polarization of the working electrode was fixed against a saturated calomel reference electrode (SCE), purchased from Radiometer Analytical S.A.

The various stages of the immunosensor build-up were interrogated by both cyclic voltammetry and impedance spectroscopy. Voltalab 80 impedance analyser from Radiometer Analytical Instrument S.A., controlled by 
computer, was used. The impedance measurements were carried out by scanning the frequency from $10^{-2}$ to $10^{5}$ $\mathrm{Hz}$, acquiring five points per decade. An excitation voltage of $5 \mathrm{mV}$ was over imposed [33].

Cyclic voltammetry and impedance experiments were carried out in the presence of a $10 \mathrm{mM} \mathrm{K}_{3}\left[\mathrm{Fe}(\mathrm{CN})_{6}\right] /$ $\mathrm{K}_{4}\left[\mathrm{Fe}(\mathrm{CN})_{6}\right](1: 1)$ mixture, in $10 \mathrm{mM}$ phosphate buffer (PBS).

\section{Results and Discussion}

As detailed in the experimental section, avidin was covalently attached to the amine terminal amine groups of a self-assembled monolayer of cysteamine spontaneously generated on clean bare gold electrode, using a homobifunctional crosslinker: glutaraldehyde. Biotinlabeled anti Human IgG antibody was anchored to the subsequent avidin derivative electrode via the formation of avidin-biotin bridge. Then BSA was used as blocking agent. Schematic of cysteamine SAM-based antibody immobilization strategy is depicted in Figure 1. The immobilization steps were evaluated following different characterization techniques.

\subsection{Cyclic Voltammetry}

Cyclic voltammogram of electroactive probes was widely used as a convenient tool to monitor the various stages of the immunosensor buildup [5] [23]. Ferri/ferrocyanide system was chosen for its stability and reversible behavior. Figure 2 shows cyclic voltammograms using $10 \mathrm{mM} \mathrm{K}_{3} \mathrm{Fe}(\mathrm{CN})_{6}{ }^{3-14-}$ as a redox probe in $\mathrm{PBS}$ at $\mathrm{pH}=$ 7.4, obtained at Au bare electrode, Au/cysteamine, Au/cysteamine/glutaraldehyde/and Au/cysteamine/glutaraldehyd/avidin. As expected, when the electrode surface is modified, the electron transfer kinetics of $\mathrm{F}_{\mathrm{e}}(\mathrm{CN})_{6}{ }^{3-/ 4-}$ is perturbed. As reported in Table 1, the stepwise assembly of cysteamine, glutaraldehyde and avidin molecules on gold electrode is accompanied by a decrease of the amperometric response of the redox probe on the electrode. In fact, at the $\mathrm{pH}=7.4$, most of the amino groups of cysteamine are almost neutral $(\mathrm{pKa}=8.27)$ [34], and the cysteamine layer exhibits practically an insulating behavior. Further modification of surface with glutaraldehyde then by avidin, led to further decrease in current of the redox couple Ip, and increase in the peak-to-peak separation $\Delta \mathrm{Ep}$. This can be ascribed to surface blockage, as well as to the increase of the insulating properties of the surface. This is consistent with the increase of electron transfer barriers introduced upon the assembly of these layers and approves that cysteamine SAMs was successfully immobilized [35] [36].

Moreover, quantitative analysis of the electrode coverage can be evaluated through the cyclic voltammetric plots [37] [38]. The surface coverage $(\theta)$ was calculated from the equation: $\theta=1-\frac{Q_{\text {bare }}}{Q_{\mathrm{SAM}}}$ [38], where $Q_{\text {bare }}$ is the charge of the bare electrode and $Q_{\text {SAM }}$ is the charge of the modified electrode by cysteamine, calculated from the area under the redox peak on the cyclic voltammograms (Figure 2). The surface coverage of the electrode after immobilization of cysteamine was found to be $33 \%$, indicative of the porosity of the generated layer. This explains why the faradaic process of oxidation and reduction of the redox couple is only partially inhibited after the deposition of the SAM layer. It is worth mentioning that the differences in the length of carbon chains of monomers may lead to distinctions in the packing of the SAMs formed by these thiols. Hence, for the alkanethiols with shorter chain length like cysteamine, the decrease of the redox peaks is lower than obtained with long chain length [39].

\subsection{Contact Angle Measurements}

Further characterization of the cysteamine self-assembled layer was performed using contact angle measurements.

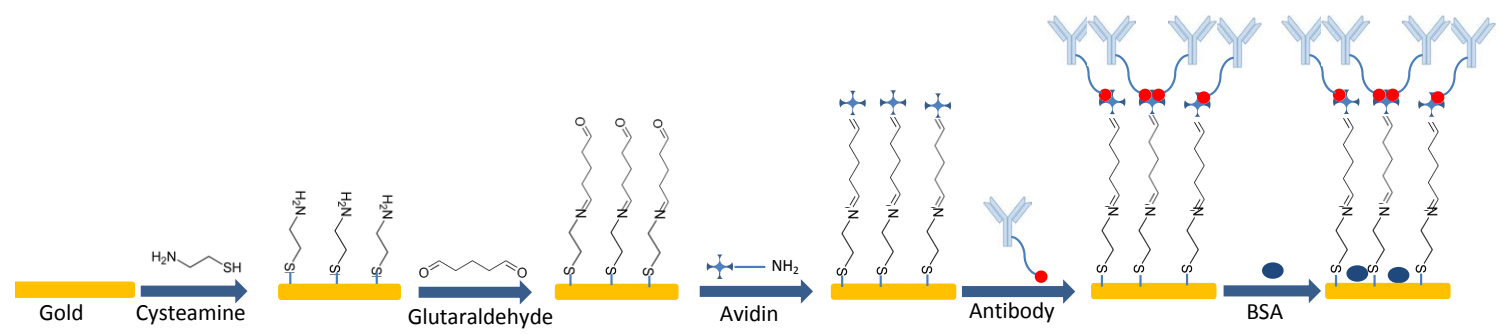

Figure 1. Illustrative scheme of cysteamine SAM-based avidin/biotin-labeled-antibody immobilization on gold surface. 


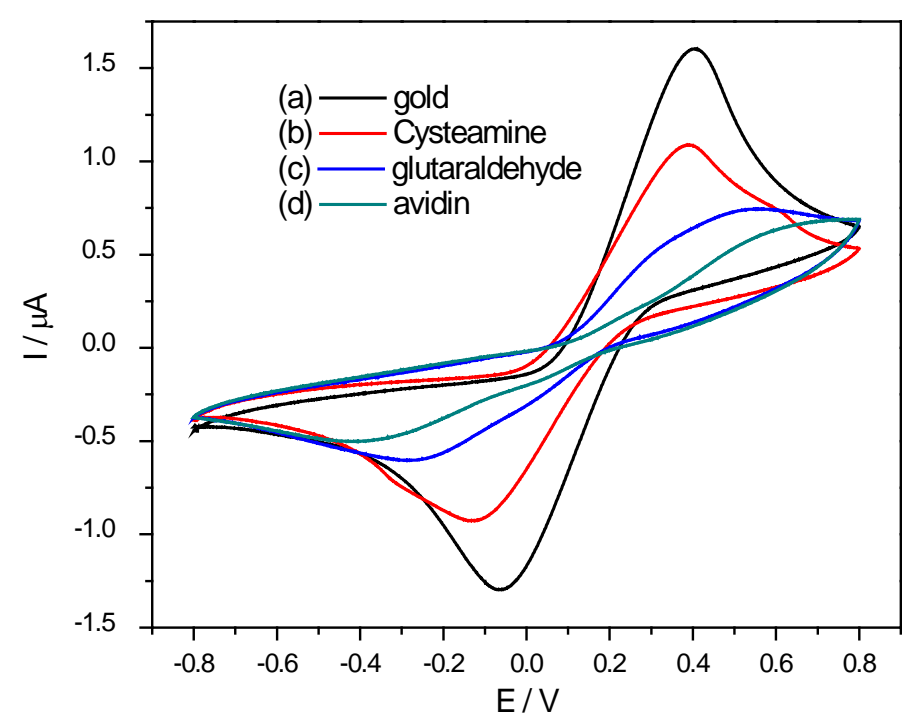

Figure 2. Cyclic voltammograms of $10 \mathrm{mM}[\mathrm{Fe}(\mathrm{CN}) 6]^{4-/ 3-}$ probe in $\mathrm{PBS}, \mathrm{pH} 7.3$, at scan rate of $100 \mathrm{mVs}^{-1}$ for: bare Au electrode (a); cysteamine/Au modified electrode (b); glutaraldehyde/cysteamine/Au modified electrode (c); and after immobilization of avidin (d).

Table 1. Characteristic values of the voltammograms of $10 \mathrm{mM}$ ferrocene in PBS extracted from curves displayed in Figure 2.

\begin{tabular}{ccccc}
\hline $\begin{array}{c}\text { Cyclic voltammetry } \\
\text { Parameters }\end{array}$ & $\mathrm{Au}$ & Electrode & Avidin \\
\cline { 2 - 5 } & 1.6 & $\mathrm{SAM}$ & 0.75 & 0.69 \\
$\mathrm{I}_{\mathrm{pa}}(\mu \mathrm{A})$ & -1.3 & 1.09 & -0.6 & -0.50 \\
$\mathrm{I}_{\mathrm{pc}}(\mu \mathrm{A})$ & 0.41 & -0.93 & 0.55 & 0.8 \\
$\mathrm{E}_{\mathrm{pa}}(\mathrm{V})$ & -0.07 & 0.39 & -0.29 & -0.43 \\
$\mathrm{E}_{\mathrm{pc}}(\mathrm{V})$ & 0.47 & -0.13 & 0.84 & 1.23 \\
$\Delta \mathrm{Ep}(\mathrm{V})$ & & 0.52 & & \\
\hline
\end{tabular}

The water contact angle for the cleaned bare gold electrode was found to be $85^{\circ}$. After the immobilization of cysteamine the contact angle decreased to $45^{\circ}$ which is consistent with the results established in literature [40]. In fact, the terminal basic amino groups of cysteamine increase the hydrophobicity of electrode surface which leads to a decrease of the contact angle with respect to the naked electrode. Hence, Contact angle measurements provide additional evidence that self-assembled monolayer was formed on the gold electrode.

\subsection{Impedance Spectroscopy}

In conjunction with cyclic voltammetry, EIS was frequently used to further characterize the stepwise modification of immunosensor [41] [42]. Initially, we have investigated the bias potential impact on the electrode-solution interface impedance. For this purpose, the impedance plots were carried out for the Au/cysteamine electrode at various bias potentials (Figure 3). The impedance measurements show typical curves for thiol self-assembled layer on metal [26] [37] [40]. For all potentials, the impedance spectra represented in the complex plane (Nyquist plots) consist of a semicircle portion at higher frequencies. When the potential is zero, a linear part appeared at low frequencies corresponding to the diffusion controlled process also known as the Warburg impedance. This behavior is attenuated with the decrease of the bias potential and vanished quasi-totally at a potential of $-1.2 \mathrm{~V}$ so that Warburg impedance was no longer observed. Thus, since antibody-antigen interaction is an interface phenomenon, it occurs also at low frequencies in the electrochemical impedance spectrum so that the diffusion phenomena could mask it. Consequently, in order to minimize the former phenomena a bias potential of $-1.2 \mathrm{~V}$ was always applied for the following investigations. Hence, the lower background impedance of the 


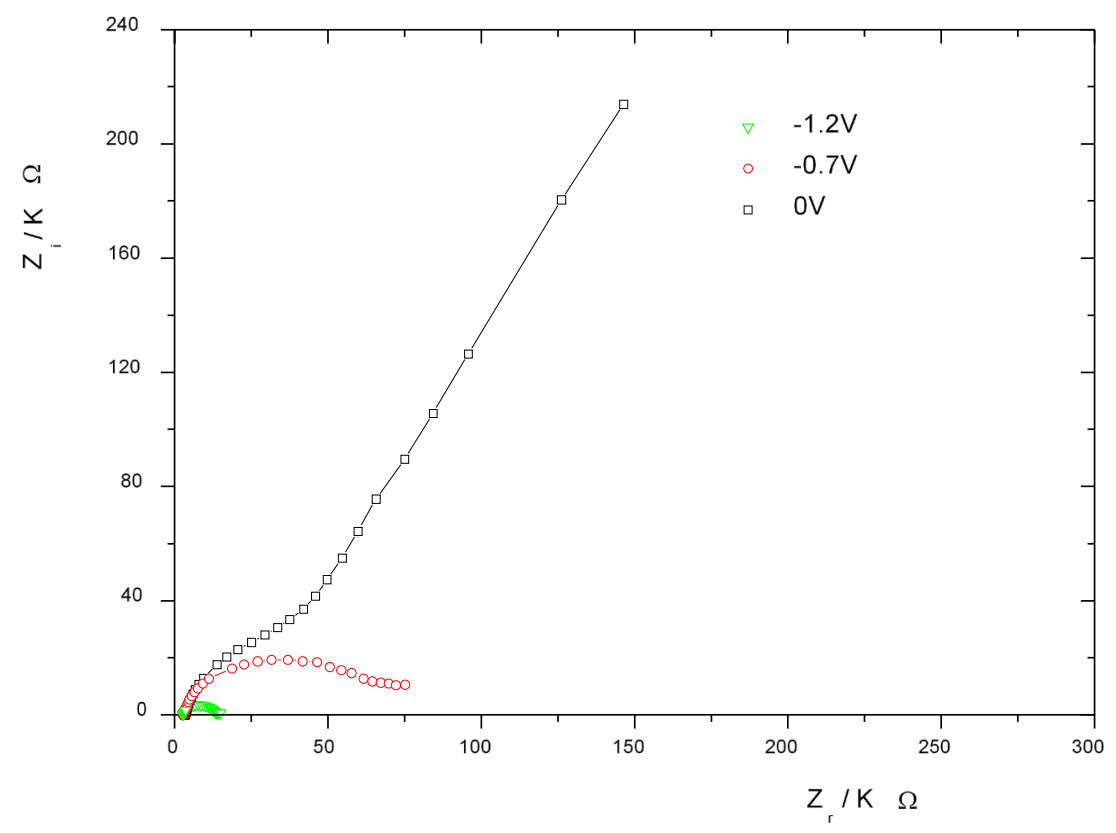

Figure 3. Nyquist diagrams for cysteamine/Au modified electrode at different poten-

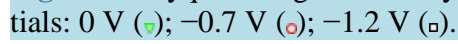

$\mathrm{Au} / \mathrm{cysteamine}$ system at this potential $(-1.2 \mathrm{~V})$ would better reveal the contribution of stepwise modification to the total impedance.

For this purpose, the impedance spectra of the cysteamine self-assembled layer, after the successive bindings of glutaraldehyde, avidin and antibody (anti-human IgG) have been recorded (Figure 4). The diameter of the semicircle was shown to increase after each step modification indicating the increase of the charge transfer resistance. These changes in the electron transfer resistance provide a strong evidence for the successful elaboration of the immunosensor starting from the SAM layer formation to antibody immobilization.

To complete the impedance investigations of the SAM layer, impedance spectrum of the cysteamine/Au electrode at a bias potential of $-1.2 \mathrm{~V}$, was fitted to an equivalent electric circuit. Although this approach is useful, where an equivalent circuit is able to mimic the behavior of the real system, the more often adopted circuits are too simplistic so that the involved electric elements are physically meaningful.

Indeed, the electrochemical system is described by an equivalent circuit involving three parts (Figure 5). The first part associated with high frequency domain includes the electrolyte resistance $\mathrm{R}_{\mathrm{s}}$ related to the bulk properties of the electrolyte solution. A second part, for intermediary frequency, attributed to the self-assembled layer, consists of parallel combination of a resistance $\mathrm{R}_{1}$ and a constant phase element $\mathrm{CPE}_{1}$. Where $\mathrm{R}_{1}$ refers to the resistance of cysteamine layer and $\mathrm{CPE}_{1}$ denotes the constant phase element substituting the capacitance of this layer and emphasizes the electrode nonidealities [7]. The third part, at low frequencies, includes in parallel combination a constant phase element $\mathrm{CPE}_{2}$ that substitutes the well-known double layer capacitance $\left(\mathrm{C}_{\mathrm{dl}}\right)$ with a charge transfer resistance $\mathrm{R}_{\mathrm{ct}}$ reflecting the easiness of electronic transfer between the bare electrode and the redox probe. Actually, The first parallel R//CPE was attributed to the electrode-film interface whereas the second was attributed to the film-solution interface. Curve fitting of the experimental data was performed with Zplot/ Zview Software (Scribner Associate Inc, Southern Pines, NC, USA). The perfect superposition between the experimental curve and the theoretical one revealed the goodness of the equivalent circuit used $\left(\chi^{2}=1.04 \times 10^{-4}\right)$ (Figure 6).

It is worth mentioning that impedimetric immunosensors cannot discriminate between specific and nonspecific interactions except through biorecognition element selectivity. Therefore, to alleviate the problem of non-specific binding causing false positive signal, the sensor surface is often pre-exposed to a solution containing a blocking agent such as bovin serum albumin (BSA) which adsorbs non-specifically reducing the eventual non-specific binding [32] [43]. Herein, in order to reduce nonspecific effects, $5 \%$ of BSA was permanently present in the buffer solution. 


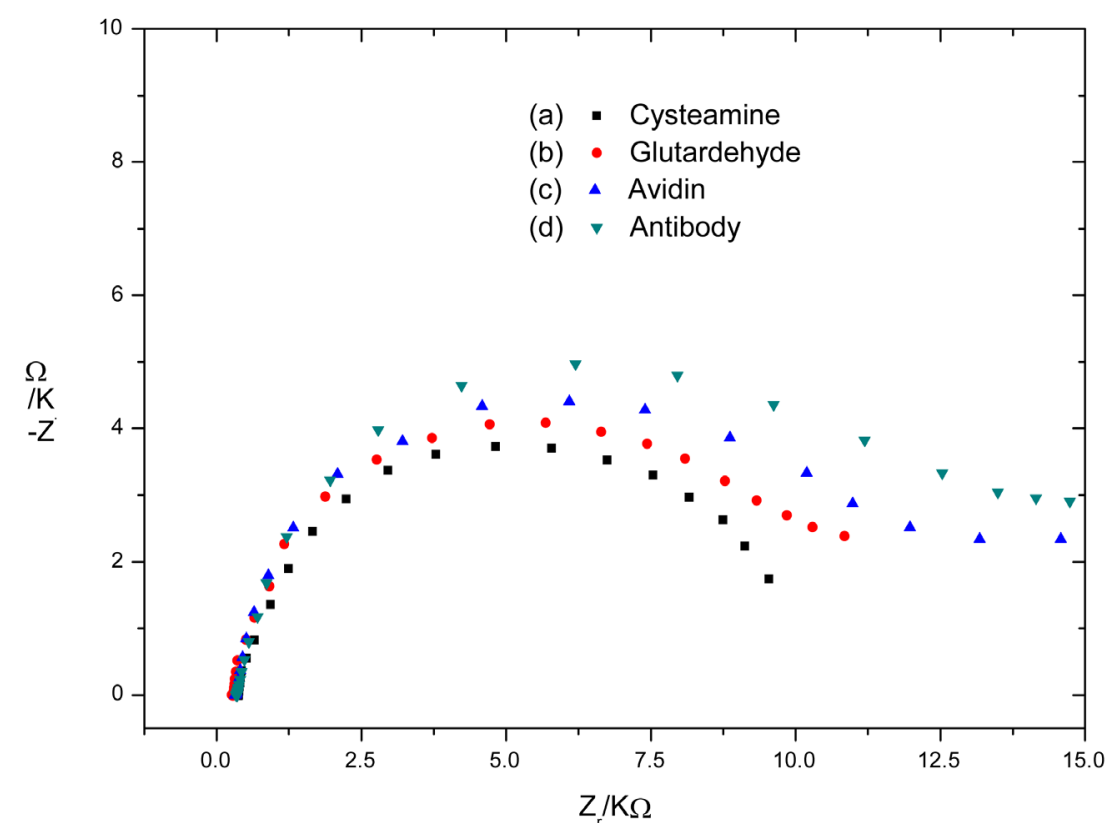

Figure 4. Nyquist diagrams at a potential of $-1.2 \mathrm{~V}$ versus SCE and ac signal of $5 \mathrm{mV}$ for: bare Au electrode (a); cysteamine/Au modified electrode; (b) glutaraldehyde/cysteamine/Au modified electrode; (c) after immobilization of avidin (d) after antibody immobilization.

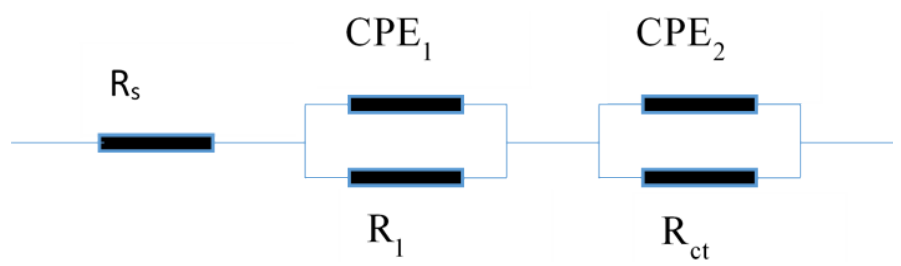

Figure 5. Equivalent electrical circuit used in the theoretical fit of the impedance.

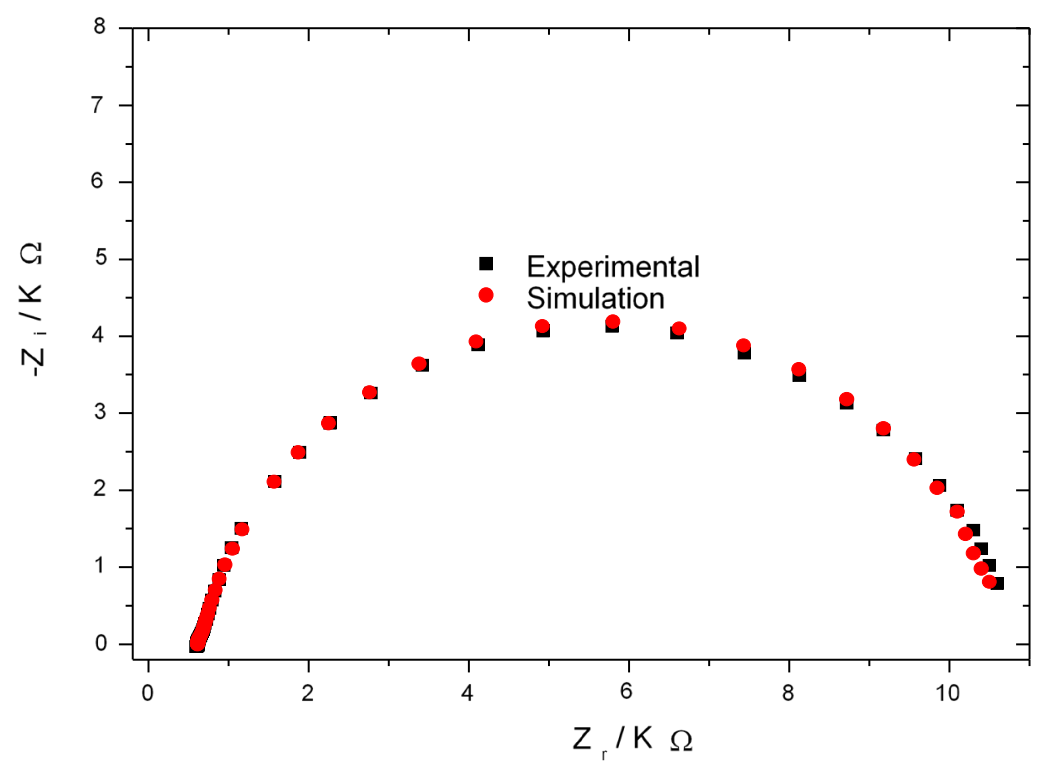

Figure 6. Experimental (₫) and calculated (๑) impedance plots obtained for cysteamine/ $\mathrm{Au}$ modified electrode at a bias potential $-1.2 \mathrm{~V}$. 


\subsection{Antigen Detection}

In order to quantify the response of the sensor, the antibody-functionalized electrode was exposed to different concentrations of antigen (human IgG reagent) and impedance measurements were performed for each concentration. As depicted on Figure 7 the increment of antigen concentration leads to gradual increase of the semicircle diameter in the Nyquist plots, typically attributed to the charge transfer resistance (Rct) of the bio-layer. Hence, the increase of the antigen concentration indicates a significant increase in the electron transfer resistance and thus increased the barrier for the electron transfer between the redox probe and the electrode surface. Alternatively, representation of the impedance spectra in the so-called Bode plan is shown. In this representation, it is obvious that the main changes in the impedance character occurred at low frequency (Figure 8).

\subsection{Calibration Curve}

Although, there is no standard method for determining immunosensor calibration curve [44], usually the changes in model elements extracted from fitting of raw impedance data to an equivalent model are reported as the sensor output [45]. Occasionally, the impedance at a particular frequency is also used. In the beginning,

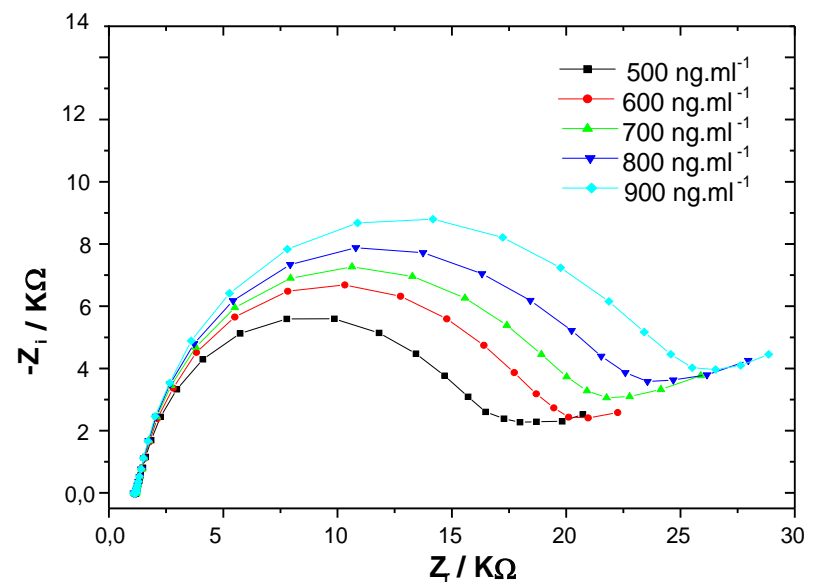

Figure 7. Complex plane impedance plots obtained for cysteamine/Au modified electrode at a potential of $-1.2 \mathrm{~V}$ versus SCE and ac signal of $5 \mathrm{mV}$, in PBS after antigen injection at different concentrations: (.): 500, (॰): 600, (^): 700, (॰):800, (): $900 \mathrm{ng} \cdot \mathrm{ml}^{-1}$ (only five curves are shown in order to avoid crowding data).

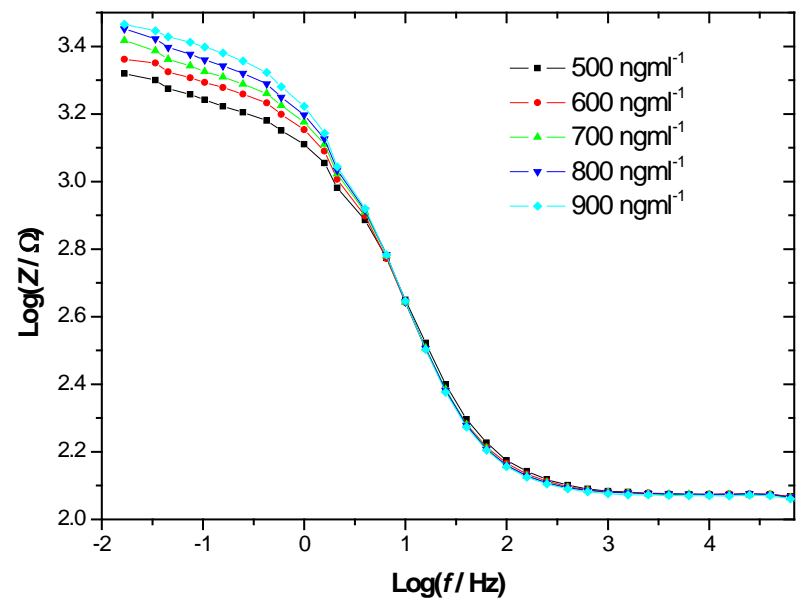

Figure 8. Bode plane impedance spectra obtained for an antibody modified electrode at a potential of $-1.2 \mathrm{~V}$ versus SCE and ac signal of $5 \mathrm{mV}$, in PBS, after antigen injection at different concentrations (.): 500, (•) 600, (॰): 700, (॰): 800, (): $900 \mathrm{ng} \cdot \mathrm{ml}^{-1}$ (only five curves are shown in order to avoid crowding data). 
since the more important change in impedance occurs at a frequency $0.1 \mathrm{~Hz}$, the latter was chosen for the study of the sensor response. So, to construct a calibration curve for the sensor, the variation of $\log \left(\mathrm{Z} / \mathrm{Z}_{0}\right)$ was plotted versus the values of the target antigen concentration at frequency of $0.1 \mathrm{~Hz}$. Where $Z_{0}$ the modulus of the impedance of the antibody modified electrode before the injection of antigens and $\mathrm{Z}$ is that of the same electrode after each antigen injection. A typical calibration curve for antigen is shown in Figure 9. This calibration curve was linear with antigen concentration from 100 to $900 \mathrm{ng} \cdot \mathrm{ml}^{-1}$, and then curved gradually at higher concentrations indicating the saturation of the specific binding sites. So, the immunosensor had a linear dynamic range from 100 to $900 \mathrm{ng} \cdot \mathrm{ml}^{-1}$ and a detection limit of about $100 \mathrm{ng} \cdot \mathrm{ml}^{-1}$.

Likewise, the impedance data were fitted by above-mentioned circuit of Figure 5 and the variation of calculated $\mathrm{CPE}_{2}$ and Rct attributed to the recognition event were plotted versus the target concentration as depicted in Figure 10. Actually, Rct controls the interfacial electron-transfer rate between the redox probe in solution and the electrode surface. As result, the growth of an insulating layer due to antigen-antibody complexes formation on the electrode surface is expected to hinder the ability of the redox probe to penetrate the electrode/electrolyte interface and therefore enhance the charge transfer resistance. As shown in Figure 10, there is a linear increase in Rct with increasing concentrations of the target antigen from $100 \mathrm{ng} \cdot \mathrm{ml}^{-1}$ to $900 \mathrm{ng} \cdot \mathrm{ml}^{-1}$. After this point a

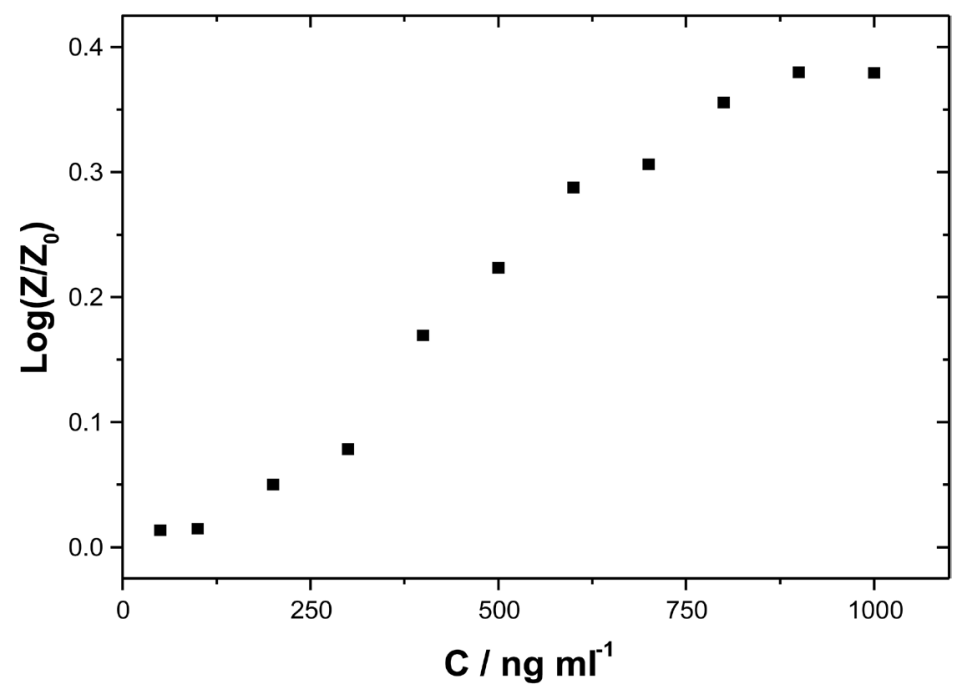

Figure 9. Response curve of the sensor, applied potential $-1.2 \mathrm{~V}$ versus SCE, frequency $0.1 \mathrm{~Hz}$, and $0.01 \mathrm{M}$ PBS (pH 7.2).

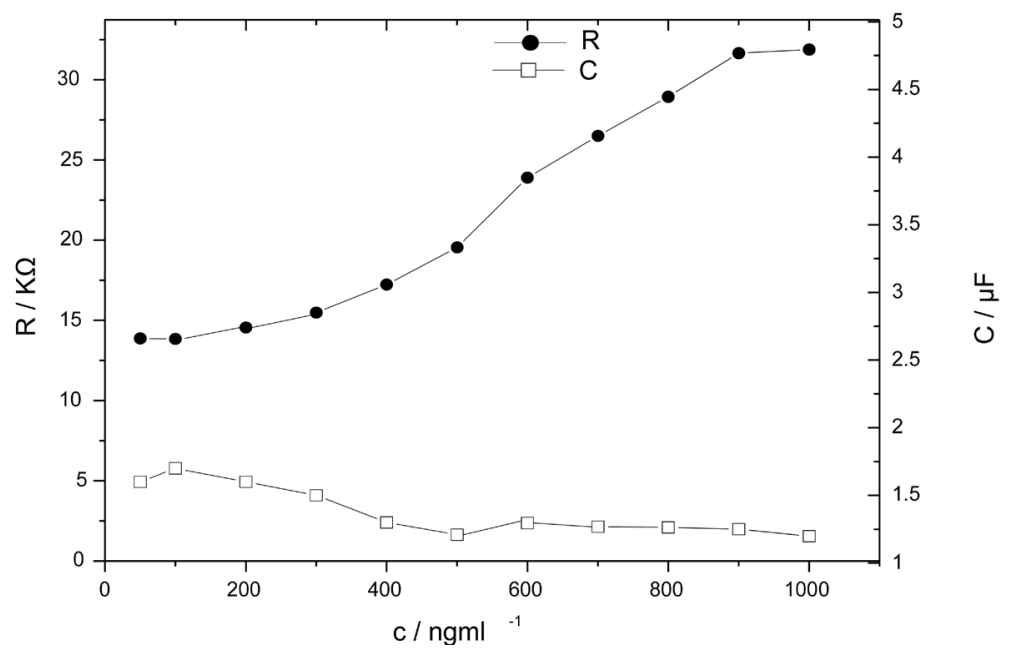

Figure 10. Variation of the calculated charge transfer resistance $\mathrm{R}_{\mathrm{ct}}$ and the constant phase element $\mathrm{CPE}_{2}$ with the antigen concentration. 
plateau was reached and a further increment of target amount did not correspond to any appreciable enhancement of the Rct. In contrast, the constant phase element $\mathrm{CPE}_{1}$ provides an insignificant decrease with the increase of the antigen concentration. In fact, since CPE is analogous to a capacitance it depends formally on the change in dielectric properties and/or thickness of the dielectric layer at the electrode-electrolyte interface following the antibody-antigen complex formation. However, the insulating layer should be thin enough and pinhole free, unless capacitance changes allocated to the binding of the analyte to the receptor might not provide significant additional change in the capacitance.

\section{Conclusion}

Avidin was, for the first time, covalently linked to a self-assembled monolayer of cysteamine generated on a bare gold electrode. By combining the strong affinity of the avidin-biotin system with the high orientation of self-assembled thiol layers, we were able to design an impedimetric immunosensor that has a wide dynamic range, from $100 \mathrm{ng} \cdot \mathrm{ml}^{-1}$ to $900 \mathrm{ng} \cdot \mathrm{ml}^{-1}$ and a very low detection limit $100 \mathrm{ng} \cdot \mathrm{ml}^{-1}$. It is expected that such combined strategy will be useful for the development of electrochemical immunosensors for the detection of other analytes.

\section{Acknowledgements}

This project was supported by the Deanship of Scientific Research at Prince Sattam Bin Abdul Aziz University under the research project 33/ص/26.

\section{References}

[1] Katz, E. and Willner, I. (2003) Probing Biomolecular Interactions at Conductive and Semiconductive Surfaces by Impedance Spectroscopy: Routes to Impedimetric Immunosensors, DNA Sensors and Enzyme Biosensors. Electroanalysis, 15, 913-947. http://dx.doi.org/10.1002/elan.200390114

[2] Guan, J.-G., Miao, Y.-Q. and Zhang, Q.-J. (2004) Impedimetric Biosensors. Journal of Bioscience \& Bioengineering, 97, 219-226. http://dx.doi.org/10.1016/S1389-1723(04)70195-4

[3] Jonathan, S.D. and Nader, P. (2007) Label-Free Impedance Biosensors: Opportunities and Challenges. Electroanalysis, 19, 1239-1257. http://dx.doi.org/10.1002/elan.200603855

[4] Mamas, I.P. (2010) Impedimetric Immunosensors. Electrochimica Acta, 55, 4227-4233. http://dx.doi.org/10.1016/j.electacta.2009.01.081

[5] Omowunmi, A.S., Austin, O.A. and Ailing, Z. (2009) Status of Biomolecular Recognition Using Electrochemical Techniques. Biosensors \& Bioelectronics, 24, 2749-2765. http://dx.doi.org/10.1016/j.bios.2008.10.003

[6] Barbero, G., Alexe-Ionescu, A.L. and Lelidis, I. (2005) Significance of Small Voltage in Impedance Spectroscopy Measurements on Electrolytic Cells. Journal of Applied Physics, 98, Article ID: 113703. http://dx.doi.org/10.1063/1.2137444

[7] Barsoukov, E. and Macdonald, J.R. (2005) Impedance Spectroscopy Theory, Experiment, and Applications. 2nd Edition, Wiley Interscience Publication, New Jersey. http://dx.doi.org/10.1002/0471716243

[8] Jung, Y., Jeong, J.Y. and Chung, B.H. (2008) Recent Advances in Immobilization Methods of Antibodies on Solid Supports. Analyst, 133, 697-701. http://dx.doi.org/10.1039/b800014j

[9] Asta, M. and Almira, R. (2013) Site-Directed Antibody Immobilization Techniques for Immunosensors. Biosensors \& Bioelectronics, 50, 460-471. http://dx.doi.org/10.1016/j.bios.2013.06.060

[10] Jaroslava, T. (1999) Oriented Immobilization of Biologically Active Proteins as a Tool for Revealing Protein Interactions and Function. Journal of Chromatography B, 722, 11-31. http://dx.doi.org/10.1016/S0378-4347(98)00434-4

[11] Ouerghi, O., Senillou, A., Jaffrezic-Renault, N., Martelet, C., Ben Ouada, H. and Cosnier, S. (2001) Gold Electrode Functionalized by Electropolymerization of a Cyano N-Substituted Pyrrole: Application to an Impedimetric Immunosensor. Journal of Electroanalytical Chemistry, 501, 62-69. http://dx.doi.org/10.1016/S0022-0728(00)00485-X

[12] Elshafey, R., Tavares, A.C., Siaj, M. and Zourob, M. (2013) Electrochemical Impedance Immunosensor Based on Gold Nanoparticles-Protein G for the Detection of Cancer Marker Epidermal Growth Factor Receptor in Human Plasma and Brain Tissue. Biosensors \& Bioelectronics, 50, 143-149. http://dx.doi.org/10.1016/j.bios.2013.05.063

[13] Wan, Y., Su, Y., Zhu, X.H., Liu, G. and Fan, C.H. (2013) Development of Electrochemical Immunosensors towards Point of Care Diagnostics. Biosensors \& Bioelectronics, 47, 1-11. http://dx.doi.org/10.1016/j.bios.2013.02.045

[14] Samanta, D. and Sarkar, A. (2011) Immobilization of Bio-Macromolecules on Self-Assembled Monolayers: Methods 
and Sensor Applications. Chemical Society Reviews, 40, 2567-2592. http://dx.doi.org/10.1039/c0cs00056f

[15] Ouerghi, O., Touhami, A., Jaffrezic-Renault, N., Martelet, C., Ouada, H.B. and Cosnier, S. (2004) Electrodeposited Biotinylated Polypyrrole as an Immobilization Method for Impedimetric Immunosensors. IEEE Sensors Journal, 4, 559-567. http://dx.doi.org/10.1109/JSEN.2004.832858

[16] Da Silva, S., Grosjean, L., Ternan, N., Mailley, P., Livache, T. and Cosnier, S. (2004) Biotinylated Polypyrrole Films: An Easy Electrochemical Approach for the Reagentless Immobilization of Bacteria on Electrode Surfaces. Bioelectrochemistry, 63, 297-301. http://dx.doi.org/10.1016/j.bioelechem.2003.09.027

[17] Cosnier, S. and Lepellec, A. (1999) Poly(pyrrole-biotin): A New Polymer for Biomolecule Grafting on Electrode Surfaces. Electrochimica Acta, 44, 1833-1836. http://dx.doi.org/10.1016/S0013-4686(98)00294-1

[18] Ballesta-Claver, J., Ametis-Cabello, J., Morales-Sanfrutos, J., Megía-Fernández, A., Valencia-Mirón, M.C., Santoyo-González, F. and Capitán-Vallvey, L.F. (2012) Electrochemiluminescent Disposable Cholesterol Biosensor Based on Avidin-Biotin Assembling with the Electroformed Luminescent Conducting Polymer Poly(luminol-biotinylated pyrrole). Analytica Chimica Acta, 754, 91-98. http://dx.doi.org/10.1016/j.aca.2012.10.006

[19] Dupont-Filliard, A., Billon, M., Livache, T. and Guillerez, S. (2004) Biotin/Avidin System for the Generation of Fully Renewable DNA Sensor Based on Biotinylated Polypyrrole Film. Analytica Chimica Acta, 515, 271-277. http://dx.doi.org/10.1016/j.aca.2004.03.072

[20] Ouerghi, O., Touhami, A., Jaffrezic-Renault, N., Martelet, C., Ben Ouada, H. and Cosnier, S. (2002) ImpedimetricImmunosensor Using Avidin-Biotin for Antibody Immobilization. Bioelectrochemistry, 56, 131-133. http://dx.doi.org/10.1016/S1567-5394(02)00029-4

[21] Wilchek, M. and Bayer, E.A. (1989) Avidin-Biotin Technology Ten Years on: Has It Lived up to Its Expectations? Trends in Biochemical Sciences, 14, 408-412. http://dx.doi.org/10.1016/0968-0004(89)90289-2

[22] Cui, X.Q., Pei, R.J., Wang, Z.X., Yang, F., Ma, Y., Dong, S.J. and Yang, X.R. (2003) Layer-by-Layer Assembly of Multilayer Films Composed of Avidin and Biotin-Labeled Antibody for Immunosensing. Biosensors \& Bioelectronics, 18, 59-67. http://dx.doi.org/10.1016/S0956-5663(02)00114-8

[23] Chaki, N.K. and Vijayamohanan, K. (2002) Self-Assembled Monolayers as a Tunable Platform for Biosensor Applications. Biosensors \& Bioelectronics, 17, 1-12. http://dx.doi.org/10.1016/S0956-5663(01)00277-9

[24] Bedford, E.E., Boujday, S., Humblot, V., Gu, F.X. and Pradier, C.-M. (2014) Effect of SAM Chain Length and Binding Functions on Protein Adsorption: $\beta$-Lactoglobulin and Apo-Transferrin on Gold. Colloids and Surfaces B: Biointerfaces, 116, 489-496. http://dx.doi.org/10.1016/j.colsurfb.2014.01.031

[25] Xue, Q.N., Bian, C., Tong, J.H., Sun, J.Z., Zhang, H. and Xia, S.H. (2011) CMOS and MEMS Based Micro Hemoglobin-A1c Biosensors Fabricated by Various Antibody Immobilization Methods. Sensors \& Actuators A: Physical, 169, 282-287. http://dx.doi.org/10.1016/j.sna.2011.01.017

[26] Hnaien, M., Diouani, M.F., Helali, S., Hafaid, I., Hassen, W.M., Renault, N.J., Ghram, A. and Abdelghani, A. (2008) Immobilization of Specific Antibody on SAM Functionalized Gold Electrode for Rabies Virus Detection by Electrochemical Impedance Spectroscopy. Biochemical Engineering Journal, 39, 443-449. http://dx.doi.org/10.1016/j.bej.2007.09.018

[27] Elshafey, R., Tlili, C., Abulrob, A., Tavares, A.C. and Zourob, M. (2013) Label-Free Impedimetric Immunosensor for Ultra-Sensitive Detection of Cancer Marker Murine Double Minute 2 in Brain Tissue. Biosensors \& Bioelectronics, 39, 220-225. http://dx.doi.org/10.1016/j.bej.2007.09.018

[28] Yabuki, S., Mizutani, F., Sato, Y. and Hirata, Y. (2003) Immobilization of Polyglutamate-Glucose Oxidase onto a Cysteamine-Modified Gold Electrode. Sensors \& Actuators B: Chemical, 91, 187-190. http://dx.doi.org/10.1016/S0925-4005(03)00086-8

[29] Akin, M., Yuksel, M., Geyik, C., Odaci, D., Bluma, A., Höpfner, T., Beutel, S., Scheper, T. and Timur, S. (2010) Alcohol Biosensing by Polyamidoamine (PAMAM)/Cysteamine/Alcohol Oxidase-Modified Gold Electrode. Biotechnology Progress, 26, 896-906. http://dx.doi.org/10.1002/btpr.372

[30] Ferretti, S., Paynter, S., Russell, D.A. and Sapsford, K.E. (2000) Self-Assembled Monolayers: A Versatile Tool for the Formulation of Bio-Surfaces. Trends in Analytical Chemistry, 19, 530-540. http://dx.doi.org/10.1016/S0165-9936(00)00032-7

[31] Zhang, Y.W., Li, Y.Q., Wu, W.J., Jiang, Y.R. and Hu, B.R. (2014) Chitosan Coated on the Layers’ Glucose Oxidase Immobilized on Cysteamine/Au Electrode for Use as Glucose Biosensor. Biosensors \& Bioelectronics, 60, 271-276. http://dx.doi.org/10.1016/j.bios.2014.04.035

[32] Poitras, C. and Tufenkji, N. (2009) A QCM-D-Based Biosensor for E. coli O157:H7 Highlighting the Relevance of the Dissipation Slope as a Transduction Signal. Biosensors \& Bioelectronics, 24, 2137-2142.

http://dx.doi.org/10.1016/j.bios.2008.11.016

[33] Darowicki, K. (1995) The Amplitude Analysis of Impedance Spectra. Electrochimica Acta, 40, 439-445. 
http://dx.doi.org/10.1016/0013-4686(94)00303-I

[34] Martell, A.E. and Smith, R.M. (1975) Critical Stability Constants. Plenum Press, New York.

[35] Hu, X.-Y., Xiao, Y. and Chen, H.-Y. (1999) Adsorption Characteristics of Fe(CN) ${ }_{6}{ }^{3-/ 4-}$ on Au Colloids as Monolayer Films on Cysteamine-Modified Gold Electrode. Journal of Electroanalytical Chemistry, 466, 26-30. http://dx.doi.org/10.1016/S0022-0728(99)00113-8

[36] Kerekovic, I., Milardovic, S., Palcic, M. and Grabaric, Z. (2014) Characterization of Cysteamine Self-Assembled on Gold Functionalized with Nitrilotriacetic Acid and Evaluation of Copper(II) Binding Capacity with Adsorption Transfer Stripping Voltammetry. Journal of Electroanalytical Chemistry, 724, 103-110. http://dx.doi.org/10.1016/j.jelechem.2014.04.017

[37] Campuzano, S., Pedrero, M., Montemayor, C., Fatás, E. and Pingarrón, J.M. (2006) Characterization of AlkanethiolSelf-Assembled Monolayers Modified Gold Electrodes by Electrochemical Impedance Spectroscopy. Journal of Electroanalytical Chemistry, 586, 112. http://dx.doi.org/10.1016/j.jelechem.2005.09.007

[38] Ramos-Jesus, J., Carvalho, K.A., Fonseca, R.A.S., Oliveira, G.G.S., Barrouin Melo, S.M., Alcântara-Neves, N.M. and Dutra, R.F. (2011) A Piezoelectric Immunosensor for Leishmania chagasi Antibodies in Canine Serum. Analytical and Bioanalytical Chemistry, 401, 917-925. http://dx.doi.org/10.1007/s00216-011-5136-7

[39] Campuzano, S., Galvez, R., Pedrero, M., De Villena, F.J.M. and Pingarron, J.M. (2002) Preparation, Characterization and Application of Alkanethiol Self-Assembled Monolayers Modified with Tetrathiafulvalene and Glucose Oxidase at a Gold Disk Electrode. Journal of Electroanalytical Chemistry, 526, 92-100. http://dx.doi.org/10.1016/S0022-0728(02)00753-2

[40] Yu, H.Z., Zhao, J.W., Wang, Y.Q., Cai, S.M. and Liu, Z.F. (1997) Fabricating an Azobenzene Self-Assembled Monolayer via Step-by-Step Surface Modification of a Cysteamine Monolayer on Gold. Journal of Electroanaiytical Chemistry, 438, 221-224. http://dx.doi.org/10.1016/S0022-0728(97)00055-7

[41] Ehret, R., Baumann, W., Brischwein, M., Schwinde, A., Stegbauer, K. and Wolf, B. (1997) Monitoring of Cellular Behaviour by Impedance Measurements on Interdigitated Electrode Structures. Biosensors \& Bioelectronics, 12, 29-41. http://dx.doi.org/10.1016/0956-5663(96)89087-7

[42] Patolsky, F., Zayats, M., Katz. E. and Willner, I. (1999) Precipitation of an Insoluble Product on Enzyme Monolayer Electrodes for Biosensor Applications: Characterization by Faradaic Impedance Spectroscopy, Cyclic Voltammetry, and Microgravimetric Quartz Crystal Microbalance Analyses. Analytical Chemistry, 71, 3171-3180. http://dx.doi.org/10.1021/ac9901541

[43] Canbaz, M.Ç., Şimşek, Ç.S. and Sezgintürk, M.K. (2014) Electrochemical Biosensor Based on Self-Assembled Monolayers Modified with Gold Nanoparticles for Detection of HER-3. Analytica Chimica Acta, 814, 31-38. http://dx.doi.org/10.1016/j.aca.2014.01.041

[44] Bart, M., Stigter, E.C.A., Stapert, H.R., De Jong, G.J. and Van Bennekom, W.P. (2005) On the Response of a Label-Free Interferon-Gamma Immunosensor Utilizing Electrochemical Impedance Spectroscopy. Biosensors \& Bioelectronics, 21, 49-59. http://dx.doi.org/10.1016/j.bios.2004.10.009

[45] Rodriguez, M.L.L., Benimeli, C., Madride, R.E. and Giacomelli, C.E. (2015) A Simple Streptomyces Spore-Based Impedimetric Biosensor to Detect Lindane Pesticide. Sensors and Actuators B: Chemical, 207, 447-454. http://dx.doi.org/10.1016/j.snb.2014.10.030 